Cad.Est.Ling., Campinas, 47(1) e (2):119-128, 2005

\title{
LA ESCRITURA DE ADULTOS Y LAS RELACIONES ENTRE EL SUJETO Y LA LENGUA
}

\author{
NORMA B. DESINANO \\ (FACUlTAD DE HuMANIDADES y ARTES \\ Universidad Nacional de Rosario - Rca. Argentina)
}

\begin{abstract}
RESUMEN Sobre la base de la fragmentariedad como una característica reiterada emergente de los trabajos escritos de estudiantes universitarios - a partir de la aparición de cortes, fallos, desorganizaciones y otros fenómenos similares-, este trabajo propone la posibilidad de recuperar el planteo del interaccionismo desarrollado como reflexión teórica por la Dra. Claudia Lemos. La propuesta en este caso se realiza en relación con la escritura de adultos. Se trata en primer término de describir los tipos de efectos relacionados con la fragmentariedad para luego relacionar estos efectos con la reflexión teórica. La argumentación gira en torno a la tesis de que la escritura del texto científico-disciplinar propone una instanciaciación a la vez. distinta y similar del sujeto capturado por la lengua. En esta instancia de escritura de un texto científicodisciplinar se percibe la interacción sujeto-lengua, poniendo de manifiesto características propias de la primera, de la segunda y de la tercera posición de acuerdo con la propuesta teórica. Se hace posible también reconocer a través del análisis un fenómeno de modificación en el uso convencional del paradigma que pone en evidencia la instancia de cambio a través de la cual cobra sentido la posibilidad tanto del cambio lingüístico como de la modificación de la relación del sujeto con la lengua.
\end{abstract}

Creo que la forma más adecuada de participar en este evento es plantear de qué modo la postura teórica desarrollada por la Dra. Claudia Lemos, abrió el camino para plantearme una hipótesis de trabajo y desarrollar una investigación, la de mi tesis doctoral, que en este momento se está cerrando, si es posible pensar que una investigación se cierra alguna vez.

El problema que me llevó a iniciar esta investigación fueron las características que presentan muchos trabajos escritos de alumnos universitarios que he debido leer y evaluar a lo largo de una larga carrera docente. Esas características, estudiadas en profundidad, no responden a patrones plausibles en relación con una de las causas que generalmente se usa para explicarlas: el supuesto desconocimiento de aspectos gramaticales y textuales de la lengua materna.

Los errores se corresponden en muchos casos con dificultades aparentemente derivados de aquella causa, pero su aparición - dentro de distintos trabajos del mismo sujeto o dentro de un mismo trabajo - no es sistemática, es decir que pueden estar presentes en un texto y no en otro, pueden ser más frecuentes en una parte de un texto y no en otras, y no responden siempre al mismo patrón o no afectan al mismo nivel del sistema. 
Como un elemento más -que dentro del corpus se fue marcando como especialmente interesante -, se planteó el descubrimiento de partes de los textos en los cuales aparecen enunciados recuperados de los textos leídos / escuchados, pero sin relación entre sí, sin una clara inserción contextual o creando ambigüedad y hasta contradicción. En muchos casos buena parte de la escritura de los alumnos muestra esta configuración de bloques aislados, como si se tratara de una acumulación de citas - no marcadas como tales - cuyas relaciones no son discernibles a primera vista, pero que constituyen una amalgama que produce un efecto visual de texto. En otros términos, uno de los rasgos notorios en muchos de estos textos es su fragmentariedad como efecto de la discontinuidad textual.

La profundización en los fundamentos de la postura teórica sobre la adquisición que ha desarrollado la Dra. Claudia Lemos, me puso sobre la pista de que muchos de los errores cometidos por los niños mientras aprenden su lengua materna participan del mismo carácter impredecible que caracterizan en gran medida a los fenómenos que acabo de señalar en los textos de adultos, en este caso textos escritos por alumnos universitarios. Del mismo modo la recuperación, a veces insólita, de enunciados fragmentarios proponía una posible relación con los primeros enunciados infantiles.

Es evidente que esta forma de mirar tanto los rasgos de fragmentariedad como los errores no surgió de manera clara y precisa en el inicio de mi trabajo sino que, más bien, el acercamiento a las explicaciones teóricas en uso, por ejemplo la del sociointeraccionismo o la del constructivismo fueron poniendo en evidencia los límites propios de estas teorías y, sobre todo, su inoperancia en el momento de señalar causas específicas en relación con problemas generales no reducibles a estándares cuantitativos ni tampoco a rasgos descriptivos cualitativamente semejantes, por lo menos dentro del marco ofrecido por el sistema de la lengua.

El acercamiento paulatino a la propuesta teórica de la Dra. Lemos, en cambio, me permitió visualizar al texto en primer lugar como fenómeno específicamente lingüístico es decir no asimilable ni explicable necesariamente en términos de una teoría general del aprendizaje-; en segundo lugar, considerar el texto como un fenómeno discursivo que pone en evidencia el dinamismo de la relación sujeto / lengua, en el que un sistema aparentemente aprehendido y aprendido - el tratarse de textos de adultos permite pensarlo así-, se torna elusivo en el momento de dar lugar a la plasmación del texto.

En otro sentido consideré fundamental tener en cuenta que la propuesta teórica a la que estaba accediendo, me permitía explicar los errores de manera consistente tanto respecto de los textos de adultos como los que aparecen en $\operatorname{los}_{\text {textos }}{ }^{1}$ de niños que están aprendiendo la lengua. Este hecho me pareció de singular importancia ya que permitía reconocer que en los fenómenos lingüísticos siempre se trata - como intentaré mostrar - de las instancias variables de la relación de los sujetos con la lengua, más allá de los límites supuestos de la adquisición.

Esto implicaría eventualmente que, a partir de la reflexión teórica de la Dra. Lemos, los avances de mi investigación podrían establecer argumentos que explicarían - en forma

${ }^{1}$ Como es obvio me refiero a textos orales en este caso y que son muchas veces solo enunciados sueltos. 
muy distinta de la utilizada hsta aquí por las teorías cognitivistas -, la persistencia y al mismo tiempo el carácter de los errores en los textos escritos de adultos.

Cabe en este punto plantear que si se pueden alcanzar estos objetivos sería también posible comenzar a reconsiderar las propuestas y las estrategias usuales dentro del campo pedagógico-didáctico en relación con la escritura. En este sentido creo que, dentro de los ámbitos escolares, cualquiera sea el nivel de enseñanza, prevalece el presupuesto de que la lengua se aprende y una vez logrado ese aprendizaje debe manifestarse en todos y en cada uno de los actos de escritura de los alumnos, y que si así no ocurre, un proceso de relectura y reescritura de los textos - convertido en monitoreo automático- puede por sí solo llevar a la superación del error.

Mi trabajo tiende justamente a examinar dos interrogantes cuya relevancia queda oculta en el planteo cognitivista, que no atiende a la persistencia de las dificultades más allá de lo que esa teoría considera como proceso de aprendizaje. Estos interrogantes son:

\begin{abstract}
por qué surge la fragmentariedad y aparecen errores en algunas partes de los textos y no en otras que aparentemente podrían crear las mismas dificultades; $\mathrm{y}$

$\gg$ por qué a veces los alumnos no parecen reconocer esa fragmentariedad ni advertir sus errores cuando efectivamente realizan operaciones de relectura y reescritura de sus textos, o incluso cuando sus pares y los profesores se los señalan.

Tomaré estosinterrogantes como eje de este trabajo e intentaré explicar por qué en todo texto pueden aparecer fenómenos lingüísticos que en términos escolares se consideran como errores y que éstos no se relacionan con instancias cognitivas, ni tampoco son resultado de circunstancias casuales y mucho menos pueden ser desestimados como escoria ${ }^{2}$, irrelevante en última instancia en el momento de analizar los textos ${ }^{3}$.
\end{abstract}

Los objetivos que marcarán el recorrido de esta presentación se relacionan entonces con :

Wexplicar, dentro de la propuesta interaccionista de adquisición, algunos de los errores lingüísticos
que se advierten en un texto escrito de un alumno universitario.
$\cdots$ permitir visualizar a través de este análisis las perspectivas de la propuesta de la Dra. Lemos en
un campo de reflexión teórica que abarca al funcionamiento lingüístico del adulto en la escritura.

He elegido especialmente dos partes muy significativas de uno de los Casos analizados en mi tesis, y que constituyen parte de una respuesta a la consigna de establecer las diferencias entre la teoría conductista y la teoría interaccionista bruneriana de adquisición del lenguaje.

El que sigue es uno de los ítema en los que se plantean las características del conductismo como teoría de adquisición:

Las tareas de aprendizaje se eligieron para construir teorías de aprendizaje, así como para asegurar que quien estudiara no tuviera conocimiento o predisposición hacia el material que se iba a estudiar.

${ }^{2}$ Tomo el término escoria en el mismo sentido en que lo hace Chomsky en Estructuras sintácticas.

${ }^{3}$ Cabe aclarar que los textos que analizo -científico-disciplinares- forman parte de una subcategoría muy especial, pero este recorte no invalida la posibilidad de generalizar algunas reflexiones que se desprendan de la investigación a otros tipos de textualizaciones. 
DESINANO - La escritura de adultos y las

La característica fundamental de este enunciado es que no presenta ningún tipo de fallas gramaticales ni relacionadas con el uso de marcas auxiliares del sistema de escritura; incluso el giro así como implica un muy buen nivel de uso de una variedad formal propia de la escritura; pero, a pesar de ello, se trata de un texto ininteligible. Me parece que por eso mismo resulta un fragmento especialmente significativo y, desde mi punto de vista, comparte con el nonsense $e^{4}$ ciertas características interesantes. En primer término aparecen dos paralelismos:

tareas de aprendizaje

teorías de aprendizaje

donde se establece un juego sonido/sentido entre teorías / tareas, a la vez que la repetición de de aprendizaje completa la simetría mencionada. El segundo tiene que ver con el ritmo y la rima:

predisposición

hacia el material

que se iba a estudiar

No parece casual que tanto en el inicio como en el final del enunciado aparezcan formas paralelísticas, como enmarcándolo, y podría hipotetizarse que se da una escritura en la que el hablante se ha dejado llevar mucho más allá de la inteligibilidad, enredado en el entrecruzamiento de cadenas que se convocan desde todos los niveles que pueden configurar los paradigmas. La textualización escapa totalmente a los límites de una interpretación disciplinar pero se advierten claramente cuáles son los motivos para que ello ocurra. Si se retoman aisladamente las construcciones, todas ellas parecen formar parte de un corpus teórico, pero su discurrir en el eje sintagmático plantea rupturas en los posibles sentidos que la interpretación va proponiendo:

\section{a) Las tareas de aprendizaje se eligieron para construir teorías de aprendizaje}

\section{b) así como para asegurar que quien estudiara no tuviera conocimiento o predisposición} hacia el material que se iba a estudiar.

de modo tal que a medida que se avanza en la lectura del texto se van desechando sentidos, ya que en ambos casos el predicado o parte de él frustra toda relación con las construcciones que actúan como sujetos y el conjunto se fragmenta como disyecta miembra. El enunciado muestra una yuxtaposición de elementos que se hallan unidos por afinidad de campo

\footnotetext{
${ }^{4}$ Aludo al nonsense como una configuración poética y lúdica que se basa en procesos metafóricos y metonímicos, del mismo tipo de los que consideraré en este punto del análisis, si bien resulta totalmente extraño dentro de los textos deseables en esta actividad escolar.
} 
Cadernos de Estudos Lingüísticos 47(1) e (2) - Jan./Dez. 2005

semántico - incluso de campo teórico-, por la organización morfosintáctica que es fuerte y compleja, así como por los efectos configuracionales del paralelismo que podríamos considerar como más primitivos, ya que son típicos de la oralidad ${ }^{5}$. Es posible afirmar entonces que ese enunciado - que es formalmente analizable-, no es interpretable, no puede dársele significación. Me parece interesante señalar que, de acuerdo con la reflexión teórica que sustenta mi análisis, el fenómeno parece muy compatible con la presencia de un sujeto en la segunda posición dentro de su funcionamiento discursivo, cuando los efectos de lengua lo llevan a proponer enunciaciones regidas por los procedimientos metafóricos y metonímicos, a partir de la convocatoria ejercida por los textos subyacentes del profesor/ bibliografía.

Los siguientes enunciados se refieren a la teoría de Bruner y aparecen encolumnados en el texto, con la configuración de ítema si bien no están enumerados. El primero que analizaré dice así:

\section{1) Hay un sistema de apoyo de la adquisición del lje que elabora la interacción entre los seres humanos.}

Para poder interpretar este texto debe plantearse como presupuesta la referencia al SAL, tal como lo expone Bruner en Acción, pensamiento y lenguaje ${ }^{6}$. Pragmáticamente conviene señalar que la forma verbal impersonal hay se aleja de las características del comentario de texto disciplinar al imponer taxativamente una afirmación teórica. Por otra parte la selección léxica en el caso de elabora resulta claramente inadecuada ya que crea una relación sintagmática que básicamente puede expresarse así :

\section{sistema elabora interacción}

en donde ni el sujeto gramatical ni el objeto aparecen como compatibles con la forma verbal que los relaciona. La recuperación de elabora, posiblemente de una cadena latente que desconocemos, crea un efecto de ruptura en el proceso metonímico que, dentro de la propuesta explicativa, se plantea como una muestra de un sujeto en una segunda posición, capturado una vez más por un efecto de lengua.

Para poder realizar un análisis más detenido de este enunciado 1) es necesario establecer su relación con 2) y 3 ) del mismo bloque:

2) Este sistema hace posible la adquisición del lje y que se desarrolle en el orden y con el ritmo adecuado.

3) Predispone a los seres humanos a interactuar comunicativamente.

${ }^{5}$ Jakobson, R. 1973. Le parallellisme grammatical et ses aspects russes. En Questions de Poétique. París: Seuil.

${ }^{6}$ Bruner, J. 1984. Acción, pensamiento y lenguaje. Madrid: Alianza. 
DESINANO - La escritura de adultos y las

Ambos enunciados actúan de la misma manera: son parafrásticos en relación con el propio discurso del hablante al mismo tiempo que amplían la información brindada por el primer enunciado. Es interesante observar cómo el enunciado 3), mediado por el 2), se constituye en una reformulación mucho más adecuada en relación con el marco conceptual teórico de referencia. No se trata entonces de ítema independientes sino de formas sutilmente parafrásticas:

- $\quad$ el primer enunciado propone un rasgo general del sistema de manera insatisfactoria, sobre todo a partir de la inadecuada selección léxica según la cual el SAL elabora la interacción entre los seres humanos.

- $\quad$ el segundo enunciado agrega un rasgo específico de dicho sistema y se constituye en un puente entre la primera y la segunda versión

- $\quad$ el tercer enunciado vuelve sobre el primero y lo reformula proponiendo otra construcción gramatical e incorporando cambios léxicos sustanciales, especialmente el cambio de elabora la interacción por predispone a los seres humanos a interactuar.

En este caso es importante señalar entonces el hecho de que hay una actividad parafrástica que actúa retroactivamente sobre el texto escrito en forma inmediatamente anterior, provocando cambios que implican movimientos en el discurso. Creo que en este caso, la tercera posición en el funcionamiento del sujeto se caracteriza ciertamente por una escucha que lo lleva a insistir con versiones que modifican la propuesta inicial. Los movimientos a los que me refiero tienen que ver con las sustituciones y continuidades habilitadas por el empleo de los procedimientos metafóricos y metonímicos.

Lo que resulta interesante es que, tratándose de escritura, el sujeto no haya eliminado las instanciaciones anteriores ${ }^{7}$. Esto puede implicar que el sujeto no advierte la magnitud real de las diferencias entre las enunciaciones parafrásticas, o bien se mantiene dentro de los cánones de la oralidad y no elimina las enunciaciones menos adecuadas. En cualquiera de los dos casos hay un claro avance hacia la tercera posición que se caracterizaría por cambios efectivos -adecuados o no- en relación con la configuración lingüístico-discursiva.

El enunciado 4) del mismo bloque se presenta como sigue -el texto mantiene la configuración gráfica que le dio el alumno-:

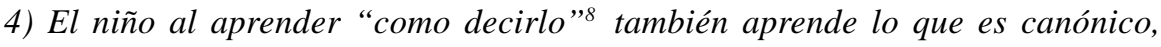
obligatorio y valorado. $\rightarrow$ esto no llega a ser lje

\section{$\downarrow$ formatos (pautas de interacción estandardizada entre adulto y niño)}

\footnotetext{
${ }^{7}$ Podría hablarse, utilizando la terminología de ciertas corrientes cognitivas, de cambios que se van produciendo "on line", pero sobre los que el sujeto no vuelve con la finalidad de ajustar el texto. Creo que es posible pensar que si bien la escucha propia de la tercera posición empieza a hacerse presente, el estudiante no ha sido en esta instancia lector de su propio texto, como lo es más adelante.

${ }^{8}$ Se trata de una fórmula interrogativa: "cómo decirlo".
} 
Cadernos de Estudos Lingüísticos 47(1) e (2) - Jan./Dez. 2005

En este caso creo que las comillas han sido utilizadas como una marca significativa destinada a establecer la denominación de algo que, en principio, el hablante no puede determinar léxicamente - así: ¿la lengua?, ¿el modelo pragmático?, ¿ los recursos comunicativos?, por ejemplo -. Queda, sin embargo en claro que "como decirlo" plantea, quizás involuntariamente por parte del hablante, una neta diferencia entre el decir y lo dicho, en cierta medida como si formaran parte de dos órdenes diferentes de cosas, que pudieran aislarse sin más.

El texto plantea también una contradicción entre el aprendizaje de lo canónico, obligatorio y valorado, que a los efectos de la interpretación sería considerado como la lengua y su uso concreto, y la observación de que esto no llega a ser lenguaje. Es probable que aquí se halle presente una cadena latente - como podría ser la que sugiero -, cuya ausencia dificulte la interpretación:

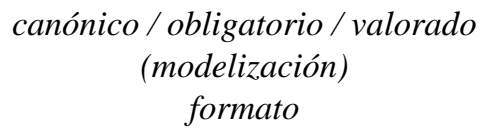

Es decir que para el sujeto que escribe, los formatos, presentados por la línea teórica como un estereotipo de actuación madre/hijo, no son una construcción de lo real que abarque los alcances de los fenómenos lingüísticos concretos. Creo que en este punto se puede advertir, en la irrupción de esto no es lenguaje, una instancia crítica a la línea teórica en desarrollo que queda solamente esbozada por la remisión a formatos que, si bien son definidos aisladamente, no son puestos en relación de contigüidad con lo que antecede. Los tres sintagmas claves no se hallan relacionados a partir de la materia lingüística: el signo gráfico, las flechas, no pueden salvar el vacío de palabras. Pero conviene advertir las resonancias de esta parte del texto en su continuidad: 
esto no llega ser lje

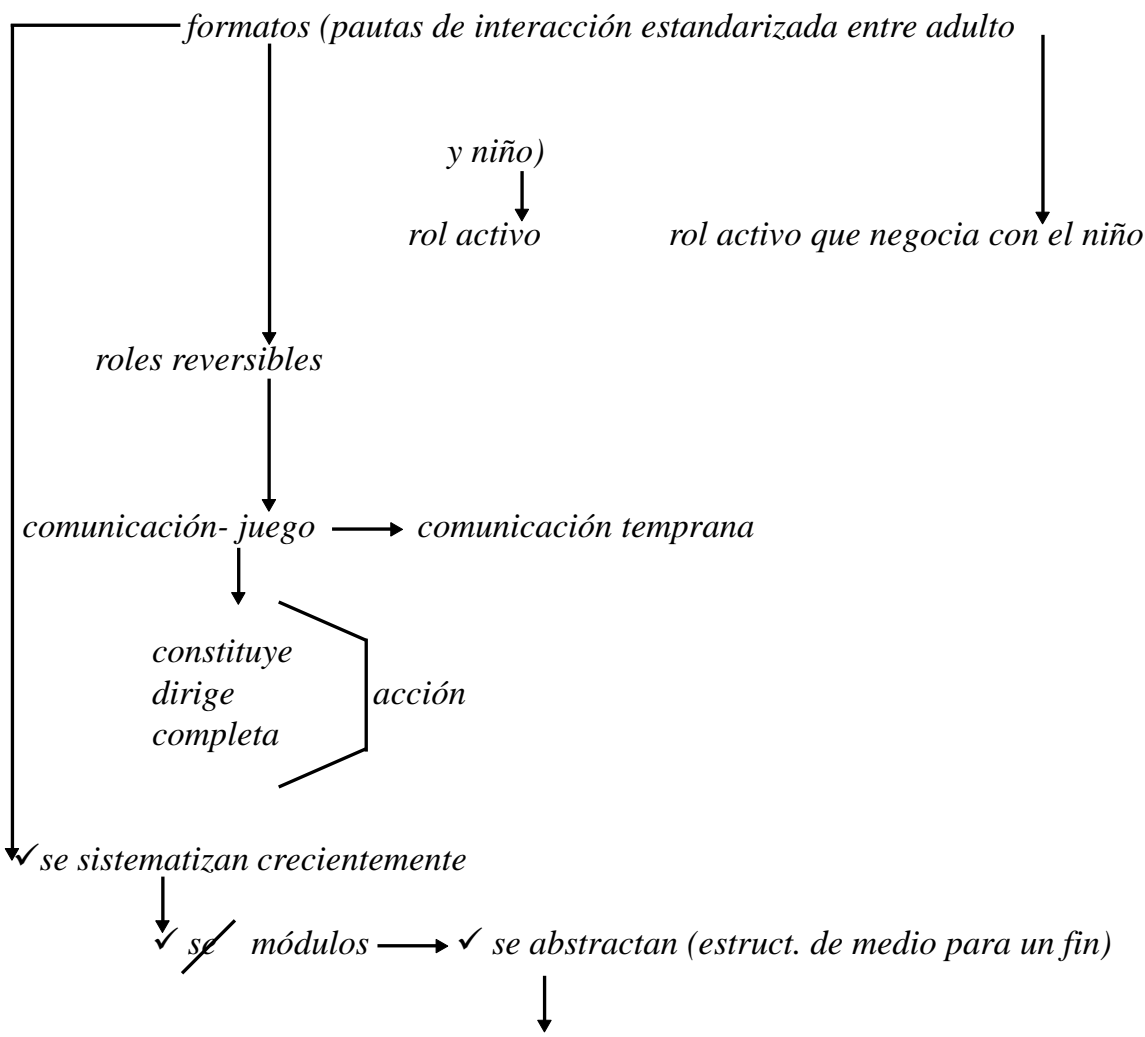

cdo. pueden ser impuestos

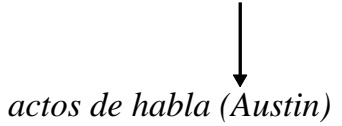

El alumno recupera aquí temáticamente formatos para realizar un desarrollo mucho más extenso. Se propone en principio una esquematización del concepto de formatos, acertada en relación con el marco de referencia teórico ya que se establecen las relaciones básicas entre acción / juego y comunicación y a partir de allí el recorrido teórico del texto del alumno plantea la sistematización creciente de formatos y una relación inteligente con los actos de habla. Si bien no realizaré el análisis de todos los aspectos que pueden resultar interesantes, me parece imprescindible volver a revisar esta parte del texto desde otro punto de vista ya que ha habido un proceso de relectura y autocorrección realizado por el alumno que tiene matices sumamente interesantes. En este sentido es evidente que se ha agregado sistemáticamente se a sistematizan, a módulos y a abstractan, suprimiendo luego el de módulos por no pertinente. Todo esto nos muestra a un sujeto en la tercera posición, 
Cadernos de Estudos Lingüísticos 47(1) e (2) - Jan./Dez. 2005

que realiza reformulaciones en muchos casos sumamente adecuadas en relación con el texto en progreso. Pero existe un rasgo que, como contraprueba, me parece que confirma el movimiento permanente de una posición a otra, tanto como la inestabilidad que los efectos de la lengua son capaces de crear, dinamizando de manera permanente el funcionamiento del sujeto en el lenguaje: ese rasgo es el empleo de la forma verbal abstractan.

Esta forma verbal aparece como una transgresión al sistema o como unaruptura paradigmática en éste, derivado del funcionamiento mismo del sujeto y no del desconocimiento por parte de este último de determinadas normas o convenciones. No se trata aquí de un trastrueque de una forma verbal, en el sentido de modificaciones desinenciales anómalas respecto de las formas canónicas, sino de algo bastante más complejo. Como puede advertirse, la primera escritura, corregida luego, decía solamente abstractan; la segunda escritura añade se. Ciertamente es posible pensar en una interpretación del tipo de se hacen o se tornan abstractos con valor cuasi-reflejo, pero así como en español son factibles léxicamente formas tales como se compactan (relacionable tanto con compacto como con campactado, formas alternativas con alto grado de relación léxica), no ocurre lo mismo con abstraer. Se abstraen se corresponde en español con abstraído, no con abstracto y el hablante, funcionando dentro de los mecanismos activo que la lengua le proporciona, produce un neologismo funcional y gramaticalmente adecuado, pero que es ajeno al sistema desde un punto de vista canónico.

Me parece importante señalar la importancia de este punto porque remite al planteo teórico que sostiene el análisis. Creo que nuevamente este efecto de lengua ha sido generado por un procedimiento metafórico que crea la extrañeza en la contigüidad sintagmática y al mismo tiempo deja en claro el modo en que el funcionamiento del sujeto en el lenguaje provoca situaciones que ponen en evidencia la posibilidad del cambio que, eventualmente, se produce o puede llegar producirse en la lengua. Se trata de un punto de inflexión en el cual la lengua y la no lengua ${ }^{9}$, podría decirse, muestran la provisoriedad de sus límites.

Es posible agregar también que este ejemplo me permite sostener una vez más la productividad de la extensión de la propuesta teórica sobre adquisición en relación con el funcionamiento adulto: como ocurre con los "errores" gramaticales de los niños pequeños, el quebrantamiento de la regla pone en evidencia un funcionamiento fluido en lo gramatical y en este caso en lo léxico. Cuando el estudiante escribe se abstractan usa una forma inadecuada al canon, pero puede usarla porque se ha establecido la interacción directa entre el sujeto y la lengua, o, si acordáramos con las teorías cognitivas, porque conoce el canon.

Esta presentación es apenas un recorte mínimo del trabajo en desarrollo, pero me parece suficientemente ilustrativo de la forma en que la propuesta teórica de la Dra. Lemos permite avanzar sobre un campo de estudio diferente al de la adquisición, que tiende, sin embargo, a corroborar las hipótesis de base ampliando su alcance.

${ }^{9}$ Milner,J.-P., 2000. El amor de la lengua. Madrid: Visor. 
DESINANO - La escritura de adultos y las

\section{BIBLIOGRAFÍA FUNDAMENTAL}

LEMOS, C. T.G. de. (1992a). Sobre o ensinar e o aprender no proceso de aquisiçao da linguagem. Cadernos de Estudos Lingüísticos 22:149-152.

(1992b). Los procesos metafóricos y metonímicos como mecanismos de cambio. Sustratum 1, pp.

121-136.

(1995). Língua e discurso na teorizaçao sobre aquisiçao de linguagem. Letras de Hoje 102, pp. 9-29. (1997). Native Speaker's Intuitions and Metalinguistics Abilities: What do they have in common from the point of View of Language Acquisition? Cadernos de Estudos Lingüísticos 33, pp. 5-14.

. (1999). A criança com(o) ponto de interrogaçao. En Lamprecht,R. (Org.) Aquisiçao de Linguagem:Questoes e Análises (pp.39-50). Porto Alegre: Edipucrs.

. (2000). Questioning the notion of development: the case of language acquisition. Culture and Psychology 6 (2), pp.169-182. 